\title{
The determination of direction in landmark-based spatial search in pigeons: A further test of the vector sum model
}

\author{
KEN CHENG \\ University of Toronto, Toronto, Ontario, Canada
}

\begin{abstract}
In four experiments, pigeons were trained to find hidden food at a constant location with respect to one or two arrays of landmarks. On crucial tests, the birds were presented with conflicting cues associated with two different directions, which were $90^{\circ}$ apart from the center of the search space at the same radial distance. The direction-averaging model predicts that the radial distance of search should not change on these tests, compared with radial distance of search on control tests without conflicting cues. The vector-averaging (vector sum) model predicts that when pigeons average the two conflicting cues, the radial distance of search should be shorter. Results support the directionaveraging model and suggest that distance and direction are independently computed in landmarkbased search. Multiple sources are averaged by pigeons in determining direction.
\end{abstract}

Many animals use visual landmarks to remember location and guide themselves back to a desired place. Some aspects of the spatial relationship between the target and surrounding landmarks are encoded and used to find the target again. Classic work by Tinbergen (1972) shows that the digger wasp uses landmarks placed around its nest to find the nest again. Tinbergen placed pine cones around a nest that a digger wasp had dug in the ground. While the wasp was away foraging, he displaced the pine cones. The digger wasp searched for her nest at the place where the displaced cones indicated that it should be. Since that study, many such landmark transformation experiments have been done to show that a variety of animals use landmarks to remember and find a place, including honeybees (Cartwright \& Collett, 1982, 1983; Dyer \& Gould, 1983; von Frisch, 1953), ants (Wehner \& Raeber, 1979), nutcrackers (Vander Wall, 1982), chickadees (Cheng \& Sherry, 1992), pigeons (Cheng, 1988, 1989, 1990; Cheng \& Sherry, 1992; Spetch, Cheng, \& Mondloch, 1992; Spetch \& Edwards, 1988), gerbils (Collett, Cartwright, \& Smith, 1986), hamsters (Etienne, Lambert, Reverdin, \& Teroni, 1993; Etienne, Teroni, Hurni, \& Portenier, 1990), and rats (Cheng, 1986; O'Keefe \& Conway, 1980; Suzuki, Augerinos, \&

This research was supported by a research grant from the Natural Sciences and Engineering Research Council of Canada to the author. I thank Helene Chevalier, Paul Miceli, and Peter Vanderheyden for help with the experimentation and data analysis, and Sylvain Fiset, Cynthia Langley, William Roberts, and Marcia Spetch for comments on earlier drafts. Portions of these results were presented at the annual meeting of the Psychonomic Society, November 1991 in San Francisco, and at the annual meetings of the Canadian Society for Brain, Behavioural, and Cognitive Sciences, June 1992 in Quebec City, and July 1993 in Toronto. Correspondence should be sent to K. Cheng, Department of Psychology, University of Toronto, Toronto, ON, Canada M5S1A1 (e-mail: cheng@psych.toronto.edu).
Black, 1980). Other examples are given by Gallistel (1990, chap. 5).

My colleagues and I have studied landmark-based spatial memory in the pigeon (Cheng, 1988, 1989, 1990; Cheng \& Sherry, 1992; Spetch et al., 1992). The first model proposed to account for the pigeon's use of landmarks was the vector sum model (Cheng, 1988, 1989). This model posits that the pigeon encodes, for each nearby landmark, the direction and distance from the landmark to the target, or a landmark-to-goal vector. In trying to find the target again, the pigeon computes, for each landmark, a navigation vector from itself to the goal (self-to-goal vector). This is done by adding the vector from itself to the landmark in question, supplied by current perception of the environment, to the landmarkto-goal vector from memory. Self-to-landmark vector plus landmark-to-goal vector equals self-to-goal vector. If a landmark has not been moved, the navigation vector associated with that landmark points to the original target location. If a landmark has been shifted, however, the target that its navigation vector points to is also shifted, in the same direction and distance as that for the landmark shift. The bird is assumed to search at the location calculated from a weighted average of all navigation vectors. This weighted average must lie on the line segment connecting the target position according to unshifted landmarks (the original goal site) and the target position according to the shifted landmark. This means that with a landmark shift, a bird may shift its peak place of searching in the direction in which the landmark had been shifted, but not in the orthogonal direction.

With a goal near a straight edge, the predictions of the model held whenever a landmark was shifted parallel or perpendicular to the edge (Cheng, 1988, 1989; Cheng \& Sherry, 1992; Spetch et al., 1992). A landmark shift in a direction diagonal to the edge, however, led to a larger 
shift of the peak place of searching parallel to the edge than perpendicular to the edge, for both pigeons and blackcapped chickadees (Cheng, 1990; Cheng \& Sherry, 1992; Spetch et al., 1992). Since the vector sum model predicts equal parallel and perpendicular shifts relative to the edge, Cheng and Sherry (1992) proposed that the birds encoded and used, in addition to vectors, the perpendicular distance from an edge to the target.

Although Cheng and Sherry's (1992) evidence suggested that the perpendicular distance to the edge was averaged in the birds' calculation of where to search, what it was averaged with was not clear. One possibility is that the landmark-to-goal vector (b in Figure 1) was used. In that case, vector averaging still takes place. But a second possibility is that the landmark-to-goal vector was decomposed into two scalars, parallel and perpendicular to the nearest edge (a and $\mathbf{c}$, respectively, in Figure 1). A compromise between the distance (scalar) c and the perpendicular distance (p) from the edge is made in determining where to search perpendicular to the edge. The scalar a determines where to search parallel to the edge. In this case, the landmark-to-goal vector is not used in the averaging process, but its component scalars are.

In this paper I present four experiments testing whether, in determining where to search, pigeons average landmarkto-goal vectors in their entirety or average the vector components of distance and direction separately. The strategy was to present tests in which two different sources of information pointed to a goal in two different directions from a landmark (Figure 2). As an example, consider Experiment 1, in which pigeons were trained to find a hidden food cup at a constant distance and direction from a cylindrical landmark. On the landmark was a strip of cardboard facing the direction of the goal. On crucial tests, the cylinder was rotated by $90^{\circ}$, turning the strip by the same amount. The bird then faced two plausible directions in which to search. One was the direction according to every landmark except the strip - that

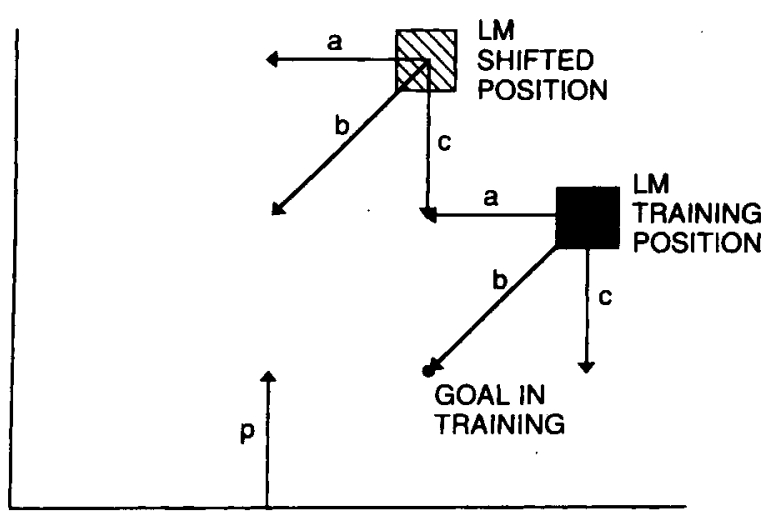

Figure 1. Theoretical possibilities for averaging when a landmark has been shifted. The perpendicular distance (p) of the goal from the edge may be averaged either with the landmark-to-goal vector $b$ or with the scalar c; $a, b$, and $c$ are all measured from the landmark, whether it is in its training position or its shifted position. See text.

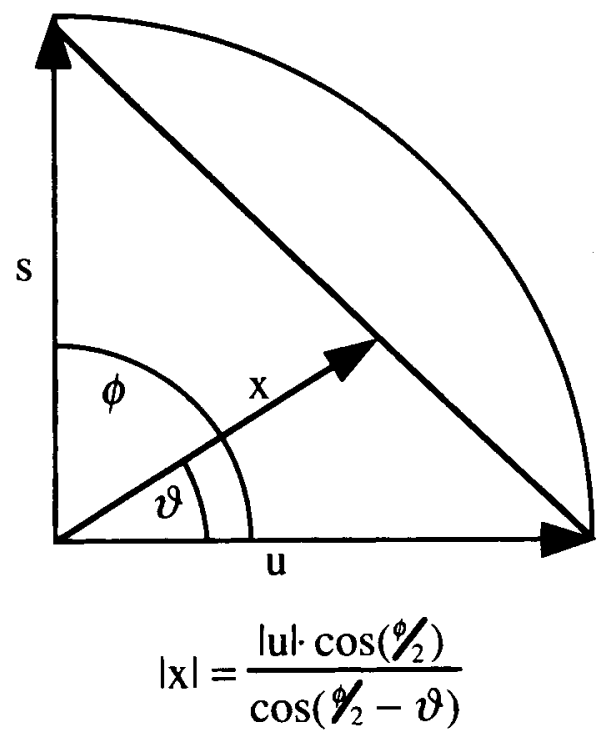

Figure 2. Predictions of the vector-averaging and direction-averaging models when two conflicting directions of search are present. The vector-averaging model predicts peak searching somewhere on the line segment connecting the endpoints of $u$ and $s$, whereas the directionaveraging model predicts peak searching somewhere on the arc connecting the endpoints of $u$ and s. See text.

is, the original unshifted direction from the landmark (u in Figure 2). The other was the direction according to the strip - the shifted direction-which was $90^{\circ}$ from the original direction ( $\mathbf{s}$ in Figure 2). The radial distance according to either vector (distance from the center of the bottle to the goal) was the same $(|\mathbf{u}|)$.

The bird presumably makes a compromise between these two theoretical points in space, but it can average in two ways. One way is to average vectors, which I call the vector-averaging model. A weighted average of two vectors must lie on the line segment connecting the endpoints of the vectors. Hence, this model predicts that the place of peak searching should lie on the line segment connecting the two endpoints of the vectors $\mathbf{u}$ and $\mathbf{s}$. A second way is to average directions and distances separately, which I call the direction-averaging model. Because $\mathbf{u}$ and $\mathbf{s}$ have the same vector length, so too is their average length, and the peak place of searching should always be at a radial distance of $|\mathbf{u}|$. The place of peak searching should thus lie on the arc connecting the two endpoints of $\mathbf{u}$ and $\mathbf{s}$, with the center as the common vector origin.

The predictions for the radial distance of search thus differ, according to the two models, when the bird searches in some intermediate direction between the two theoretical directions. The predicted radial distance of the vectoraveraging model depends on the search direction and the extent of landmark rotation; it is given by the formula in Figure 2. In contrast, the predicted radial distance of search for the direction-averaging model is always $|\mathbf{u}|$. The difference between the predictions of the two models is largest at a direction midway between $\mathbf{s}$ and $\mathbf{u}$, and it decreases when the search direction nears either of the 
directions. The two models make the same prediction of $|\mathbf{u}|$ when the search direction equals the direction of $\mathbf{s}$ or u. Since any single experiment does not generate enough data points to differentiate between the two models, I pooled the data from all the experiments before determining which model fares better.

\section{EXPERIMENT 1}

In this experiment, a single cylindrical bottle served as a landmark. Vertically placed on the bottle was a thin black cardboard strip. During training, this strip always faced the same direction and pointed to the goal. The landmark and goal array were centrally placed in a room that was completely covered with wood chips, which served as the search space. On occasional tests without the goal or food, the bottle was either in its usual orientation (control test) or was rotated by $90^{\circ}$ (experimental test). On experimental tests, the direction of the goal according to the local landmark (the strip on the bottle) was at $90^{\circ}$ variance with the direction according to other (e.g., distal) landmarks in the room.

\section{Method}

\section{Subjects}

Four White King pigeons (Columba livia) obtained from Palmetto pigeon farm (Sumter, SC) served as subjects. They had participated in experiments in traditional operant boxes, but were naive to the experimental room and the search task. The birds were reduced to between $83 \%$ and $87 \%$ of their free-feeding weights and were housed in individual cages on a 14:10-h light:dark cycle. Water and grit were available at all times in their home cages, although not in the experimental room. Experimentation took place just before they were fed their daily ration of mixed grains.

\section{Apparatus}

Chamber. The experimental chamber (Figure 3) was a room measuring $428 \mathrm{~cm} \times 262 \mathrm{~cm}$. A video camera was positioned on the center of the ceiling. The landmark arrays presented to the birds were also centrally located, within the camera's view, and were relatively far from each of the walls. At one end of the room was a door with an observation window through which the birds were observed. The walls were uniformly white and, on all but the top wall, rectangular outlines of concrete blocks were visible. The left and top walls contained electrical outlets. Overhead fluorescent lights provided lighting. The stimulus array is drawn from the viewpoint of the observer at the door and was thus oriented in the same way as that shown in Figure 3. Following the conventions of Cheng and Sherry (1992), I will call the directions, as they appear in Figure 3, up, down, left, and right.

Landmark array. Centrally located on the floor of the room was a $122 \times 122 \times 0.3 \mathrm{~cm}$ (thick) masonite board. At the center of the board were nine pieces of velcro glued in a $3 \times 3$ grid, $12.5 \mathrm{~cm}$ between the centers of neighboring pieces. These served as the possible locations of the goal, which was a bottle cap $4.5 \mathrm{~cm}$ in diameter and $1 \mathrm{~cm}$ deep. The goal was held at one of the nine locations by velcro. Most of the floor was covered with wood chips, approximately $2 \mathrm{~cm}$ deep. The area near the door was left bare. The only landmark near the goal was a cylindrical bottle, $9 \mathrm{~cm}$ in diameter at the base and $26.3 \mathrm{~cm}$ high, filled with clear fluid and tapered toward the top. A strip of black cardboard, $2 \mathrm{~cm}$ wide and $22 \mathrm{~cm}$ high, was taped along the length of the bottle. The right

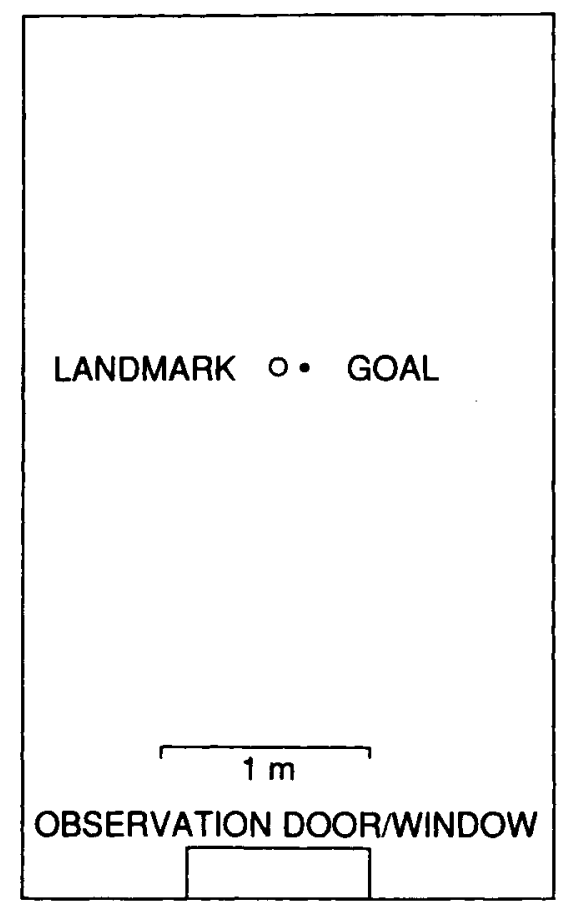

Figure 3. The experimental chamber for Experiment 1. The filled dot represents the position of the goal, and the unfilled circle represents the landmark - a bottle with a strip of cardboard pasted on it.

edge of the bottle was $13.5 \mathrm{~cm}$ left of the center of the goal. The strip on the bottle faced the goal.

\section{Procedure}

Training. The birds were individually trained by gradual shaping, following the routines from past experiments (e.g., Cheng, 1989; Cheng \& Sherry, 1992). The reward consisted of large grains (corn and various beans) from the mixed grains that served as the daily food for the birds. Only large grains were used because birds sometimes scatter food in searching. In such cases, large grains are readily found, which minimizes the risk of food left inadvertently outside of the goal. Four grains served as the standard reward, although larger amounts were provided in the beginning when the birds were being familiarized with the room.

During training, the goal location across trials was the same on each day. Across days, a different one of the nine possible goal locations was chosen. Food was first left exposed in the goal cup, and the bird was left in the room to find and eat the food. Training began when the bird approached readily to eat the food. Over trials and days, conditions approached the training situation in the following steps: (1) Four grains were left exposed in an exposed food cup; (2) some of the four grains were covered but the food cup was exposed; (3) all four grains were covered but the food cup was exposed; (4) all grains were covered, the food cup was exposed, and the landmark was in place; (5) all grains were covered, the food cup was partially covered, and the landmark was in place; and (6) the landmark was in place, and the substrate uniformly covered the food and the food cup (training). After each trial, the lights went off and the bird was covered with an opaque cardboard box. (A 7-W light and the light from the monitor in the observation room adjoining the experimental room was sufficient to see the bird.) The birds typically wandered to one of the corners, but 
they did show some variation from trial to trial. After the necessary arrangements for the next trial, the bird was released with the room lights off.

Testing. The testing phase began with 6 days in which six training trials and no test trials were presented. After that, each day consisted of four training trials and one test, which appeared on Trial 3,4 , or 5 , chosen at random. Out of the nine possible locations, the location of the goal on the first training trial was chosen at random. Thereafter, it was moved every one or two trials to an adjacent location $12.5 \mathrm{~cm}$ left, right, up, or down. The bottle, of course, moved with the goal to stand in the same position with respect to the goal from trial to trial. Two types of tests were given, which differed only in the arrangement of the landmark. Six tests of each type were given to each bird. On a control test, the bottle serving as landmark was in its usual orientation. On an experimental test, the bottle was rotated by $90^{\circ}$ counterclockwise, so that the strip faced up rather than right. On both control and experimental tests, the food cup and food were absent. The landmark was placed so that the location of the goal, with respect to the landmark (ignoring the strip), was within the grid of nine velcro pieces but not at a velcro piece. Tests were videotaped from above on a Panasonic (Seacaucus, NJ) 6030 videocassette recorder for $1 \mathrm{~min}$ from the first peck, after which the lights were turned off and the test was terminated. Before each test, a plus-shaped calibration marker, indicating the location of the goal and $7.5 \mathrm{~cm}$ in each principal direction (up, down, left, right), was videotaped for a few seconds to provide calibration of distances and locations for analyzing the search behavior.

\section{Data Analysis}

Tests were analyzed by methods similar to those used by Cheng and Sherry (1992). The video of each test was analyzed frame by frame. The recorder was put on still at the first peck, at which time an acetate sheet was laid over the monitor and calibration positions were marked on the sheet. The head position (forwardmost point) of the bird was marked every six frames $(0.2 \mathrm{sec}$ real time $)$ for a maximum of 100 marks ( $20 \mathrm{sec}$ real time). Head positions were not marked if the bird lifted its head and walked in the middle of a test. Marking resumed at the next peck. In this way, only the first $20 \mathrm{sec}$ of searching on a 60 -sec test were analyzed. The birds tend to scatter their search after awhile, and this way of scoring tests is an attempt to minimize the inclusion of this behavior.

The acetate sheet was then laid on a grid of $0.5-\mathrm{cm}$ squares (corresponding to approximately $1.3 \mathrm{~cm}$ real distance), with the goal at an intersection. The number of marks in each square were counted, and row and column totals were obtained. Rows and columns from tests of the same type were added for each bird separately. These distributions were used to obtain a peak place of search along each of the up-down and left-right dimensions. The calculation used a formal procedure, which has been used in other studies (e.g., Cheng \& Sherry, 1992). First, the median of the distribution was calculated. In calculating the median, the distribution within each bin was assumed to be flat. To ensure that the median represented the middle point of the highest region, the median calculation was iterated over a region in which the first median was the middle. Thus, if the distribution ranged from -20 to $20 \mathrm{~cm}$ and the first median was $10 \mathrm{~cm}$, the next calculation was done from 0 to $20 \mathrm{~cm}$. This process was continued until two iterated medians differed by less than $0.05 \mathrm{~cm}$. The value of the last median was taken to be the peak place along that dimension. Justification for this procedure has been given in Cheng (1989) and Roberts (1981).

Throughout the present paper, within-subjects analyses of variance (ANOVAs) are used to compare the data on control versus experimental conditions. The significance level was set at .05 .

\section{Results and Discussion}

Places of peak searching are shown in Figure 4. One bird showed substantial changes in the direction of

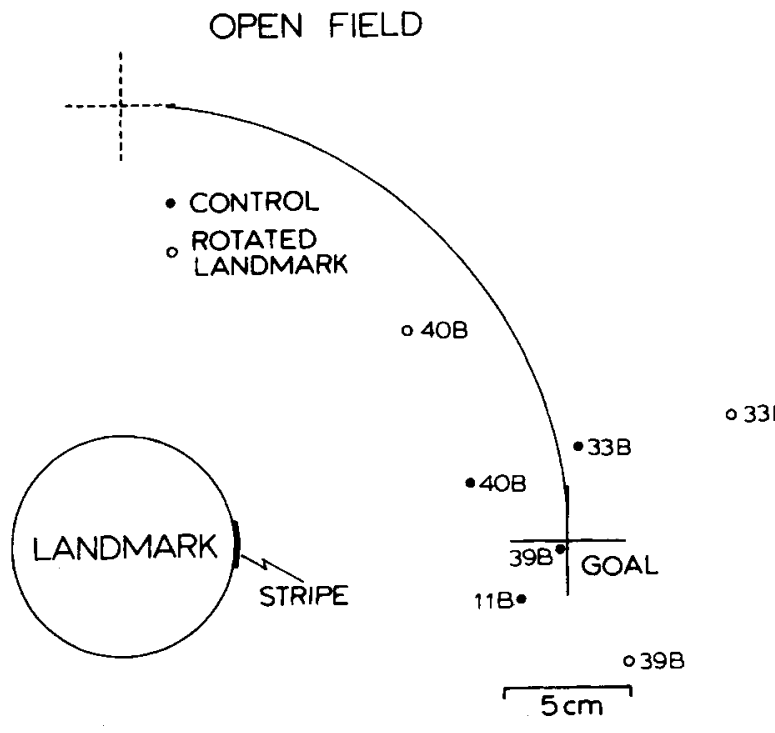

Figure 4. Places of peak searching for individual birds in Experiment 1 . The dashed cross indicates the goal location according to the rotated strip of cardboard.

search in the experimental tests with the rotated landmark. The radial distance of search for this bird (40B) was approximately equal in the control and experimental conditions - results that are in line with the directionaveraging model rather than the vector-averaging model. One other bird (11B) showed little searching scattered over a large area on tests with the bottle rotated, so its data from the experimental condition are not presented. On the whole, neither the mean direction of search nor the mean radial distance of search differed significantly between the two conditions.

Although these data show that, on the whole, the compass orientation of a strip of cardboard on a nearby landmark had little influence on the pigeons in the direction of their search, another unpublished pilot experiment showed positive evidence for the influence of the strip. The pilot experiment was carried out on 4 other pigeons of the same species. The training and testing took place on a circular tray, and the goal was a $30^{\circ}$ sector facing the strip. This meant that radial distance was rendered irrelevant, because search at any radial distance was correct given the correct direction of search. For this reason, the experiment is not reported in full here. On tests, the bottle was rotated by $0^{\circ}, 45^{\circ}$, or $90^{\circ}$. The birds showed a significant shift in the direction of the rotated landmark in that experiment and shifted to similar extents in the $45^{\circ}$ and $90^{\circ}$ bottle rotations.

\section{EXPERIMENT 2}

In Experiment 2, an attempt was made to make the bottle rotation more influential in the present procedure. Birds were trained as in Experiment 1, except that the space in which they searched was delimited by a circular tray; wood chips were found only in the tray. After the 
birds had learned the task, the entire tray was rotated by $90^{\circ}$, changing the direction of the goal as well as that of the bottle. While learning this new arrangement, the birds should show some directional conflict between the direction for the new goal location and the direction for the old goal location. If so, it was expected that they would show some searching in an intermediate direction. To differentiate between the two models, a further attempt to select data was made in that the only tests analyzed were those in the rotated second phase that showed an intermediate average direction of search.

\section{Method}

\section{Subjects}

Four White King pigeons participated; they were kept in the same conditions as those in Experiment 1. One bird (41B) was naive at the task. The other 3 had just participated in an experiment in the experimental chamber, in which for 40 sessions they repeatedly (eight times per session) approached the experimental tray for uncovered food. The tray and position of food were those used in this experiment, and the birds were familiar with the bottle used in this experiment.

\begin{abstract}
Apparatus
The search space was different from that used in Experiment 1. Wood chips were removed from the room. A circular tray $(41 \mathrm{~cm}$ in diameter, $2 \mathrm{~cm}$ high) filled with wood chips was used as the search space. It was centrally placed under the camera. Four black marks were drawn on the floor at the circumference of the tray, in the four cardinal directions; these served as calibration markers (and also as possible landmarks for the birds). The same bottle with the strip served as the landmark. It was placed at the center of the tray, with the strip facing right. The goal was smaller $(4 \mathrm{~cm}$ in diameter, $1 \mathrm{~cm}$ high). It was attached by velcro, $11 \mathrm{~cm}$ to the right of the bottle and $5 \mathrm{~cm}$ from the edge of the tray (measurements from the center of the goal).
\end{abstract}

\section{Procedure}

The experiment consisted of two phases. In Phase 1, the control phase, the strip on the bottle faced right and the goal was to its right. After initial training to search for hidden food (cf. Experiment 1 ), the birds were each trained for five sessions, eight trials per session, with wood chips uniformly covering the food and food cup on all trials. They were then tested for six sessions. Each test session consisted of five trials, onè of which was a control test that appeared at random on Trial 3, 4, or 5. On the control test, the landmark arrangement was identical to the training arrangement, but as in Experiment 1, the food and food cup were absent.

Phase 2, the experimental phase, consisted of 10 sessions of five trials each. The training arrangement was rotated $90^{\circ}$ clockwise so that the goal and the strip on the bottle faced down. Each-session consisted of four training trials and one test trial. For the first four sessions, the food cup was left uncovered on training trials, although the food in the cup was covered. For the last six sessions, the food and food cup were uniformly covered by wood chips. A test was presented at random on Trial 4 or 5 . The landmark arrangement on a test was identical to that of the new training arrangement, and the strip faced down. Otherwise, the test procedure was as usual.

\section{Data Analysis}

Data analysis followed Experiment 1 up to the marking of the acetate sheet. The sheet was placed over a circular graph paper and the data were counted circularly. The total counts in each $10^{\circ} \mathrm{sec}-$ tor were obtained, with the goal at a line separating two sectors, as were the counts in each radial ring of $0.5 \mathrm{~cm}$ (approximately $0.67 \mathrm{~cm}$ real distance). Peak place was calculated separately for different birds and test types along the radial and angular dimensions. Radially, the median iteration procedure of Experiment 1 was used. Angularly, the direction of the mean vector (Batschelet, 1981) of the distribution was the peak place. The mean vector was obtained by considering each mark as a point on the circumference of a unit circle (thus ignoring the radial distance of the mark). In this case, direction was coded to the nearest $10^{\circ}$, since the data were coded in $10^{\circ}$ sectors. The vectors from the center to each point were averaged to give the mean vector. In averaging vectors, all of the vectors were added and then divided by the number of vectors, much like averaging the scalars. The direction of the mean vector is a measure of the central tendency in the direction of the marks from the center of the tray.

Only a selection of tests from the experimental phase were included for analysis. The mean vector from individual tests of individual birds in the experimental phase was first calculated and compared with the mean vector from the pooled control tests of that bird. Only experimental tests with mean vectors between $20^{\circ}$ and $70^{\circ}$ clockwise from the mean vector of control tests were included for analysis. In this way, only tests with an intermediate direction of searching were included for analysis. Two to seven tests were selected from each bird; these were pooled and scored in the usual manner.

\section{Results and Discussion}

Places of peak searching are shown in Figure 5. On the selected experimental tests, the birds' direction of search was between $48^{\circ}$ and $63^{\circ}$ clockwise from the direction found on control (Phase 1) tests; the two conditions differed significantly $[F(1,3)=355.4]$. The radial distance of search, however, was similar (not statistically different) in the two phases. The latter results, then, support the direction-averaging model and not the vector-averaging model.

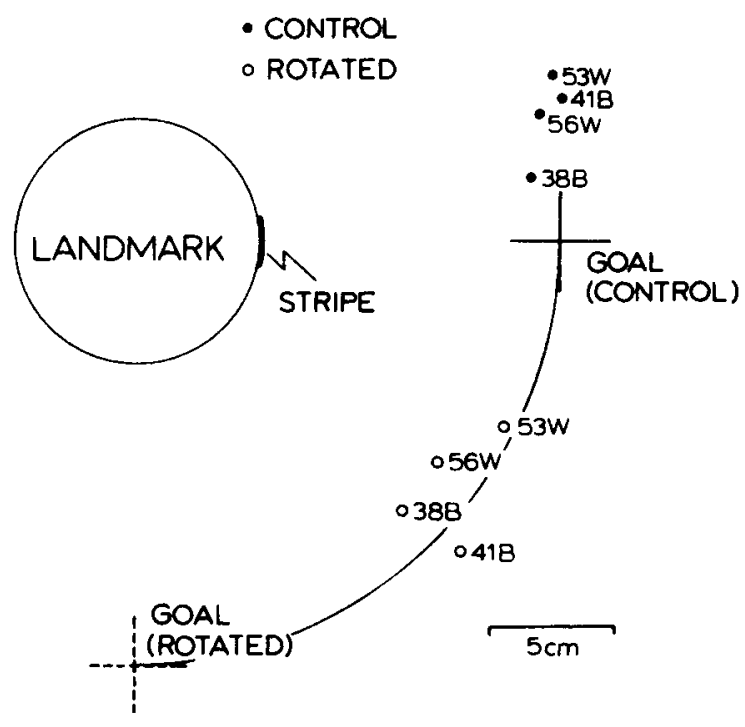

Figure 5. Places of peak searching for individual birds in Experiment 2. The dashed cross indicates the goal location according to the training arrangement in Phase 2, and the solid cross indicates the goal location according to the training arrangement in Phase 1. 


\section{EXPERIMENT 3}

In Experiment 3, a two-bottle training arrangement was used. The two cylindrical bottles differed in shape, size, and color and were placed $90^{\circ}$ apart from the goal (Figure 6). During training and on control tests, the goal was located down from one bottle landmark and to the left of the other. On experimental tests, only a single bottle was present. To search in the right direction from this single landmark, the bird had to discriminate it from the other, missing landmark and pick out the appropriate direction and distance. If the process of landmark discrimination or picking out the correct vector associated with the tested landmark fails, directional conflict is generated. For example, although the goal is down from the red landmark, if the red landmark is confused with the green one, the bird will tend to search in a leftward direction on trials with the red bottle alone. It is possible that the bird will make a compromise between these two directions, in which case the vector-averaging model would predict a radial distance of search that was shorter than that on control tests, whereas the direction-averaging model would predict the same radial distance of search on both control and experimental tests.

\section{Method}

\section{Subjects}

Four pigeons experienced at searching for hidden food participated. They had not been subjects in any of the other experiments reported here, and they were kept under the same conditions as the birds in the previous experiments.

\section{Apparatus}

The training arrangement is shown in Figure 6. The goal was the same size as in Experiment 1. The red landmark was a roughly cylindrical bottle, $8 \mathrm{~cm}$ in diameter at its widest point and $18 \mathrm{~cm}$ high. It was capped with a red cap and filled with red fluid. The green landmark was a cylindrical bottle that was tapered at the top, $9 \mathrm{~cm}$ in diameter at its base and $19 \mathrm{~cm}$ tall. It was filled with green fluid. The center of the goal was $10 \mathrm{~cm}$ from the nearest edge of each of the landmarks. The entire array was shifted about from trial to trial, as in Experiment 1.

\section{Procedure}

The birds were individually trained to search for hidden food, as in the previous experiments. They were then given 5 formal training days, five trials per day, before testing began.

On test days, one trial from Trial 3,4, or 5 was a test trial in which the goal and food were absent. Three types of tests were given, each six times. On a control test, the arrangement resembled the training condition. On a red landmark test, only the red landmark was present. On a green landmark test, only the green landmark was present. Early in testing, I piloted a number of tests in which the size of the red landmark was changed. The birds, however, showed fear of the new landmarks and produced scattered searching. These tests were not analyzed. Data analysis followed Experiment 1.

\section{Results and Discussion}

Places of peak searching are shown in Figure 6. On control tests, the birds searched in the approximate area of the goal, but on experimental tests in which only a single landmark was present, they sometimes searched at a different place. In these instances, the direction of search was rotated toward the direction appropriate for the ab-

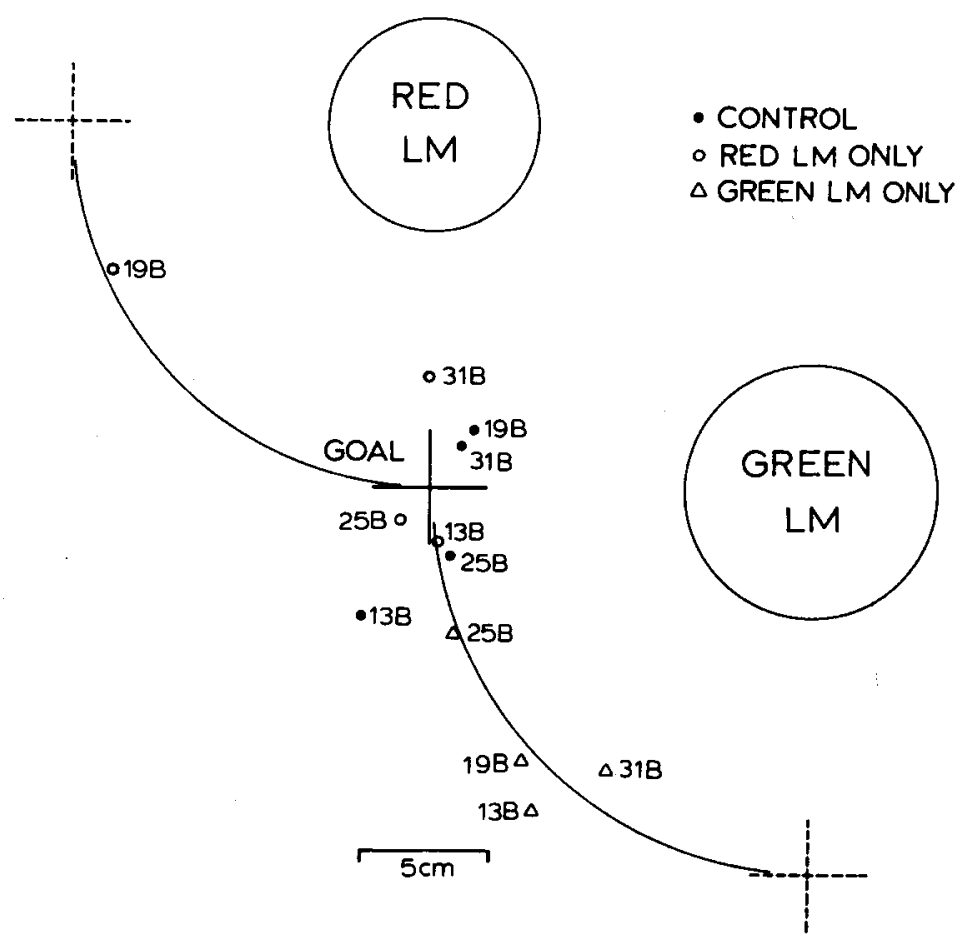

Figure 6. Places of peak searching for individual birds in Experiment 3. The dashed crosses indicate the location of the goal on single-landmark tests according to the dictates of the absent landmark. 
sent landmark. The directions of search from the green bottle were significantly different for the control and green-bottle tests $[F(1,3)=12.97]$, but the control and red-bottle tests did not differ in the direction of search from the red bottle. More importantly, the radial distance of search on the red-bottle tests did not differ significantly from the radial distance of search on the control tests, and the radial distance of search on the green-bottle tests did not differ significantly from the radial distance of search from the green bottle on control tests. These results support the direction-averaging model and contradict the vector-averaging model.

In some cases, the pigeons searched in the appropriate location even with only a single landmark. This means that the birds must have encoded the vector from individual landmarks to the goal. They may also have encoded the location of the goal with respect to the configuration of the two bottles; the data do not address this hypothesis.

The errors made by the birds on tests with single landmarks are also interesting. The birds showed a single peak of search at a place intermediate between two theoretical locations: the correct location with respect to the landmark found on the test, and the correct location with respect to the absent landmark. None of the birds showed a double peak at these two theoretical locations. The birds had thus averaged the two locations in some fashion in their searching. The main question, of course, is whether the averaging was one of directions or of vectors, although the data seem to favor the former.

\section{EXPERIMENT 4}

Another way to test whether birds encode and use a single vector from a single landmark to the goal is to train and test them with single landmarks. In Experiment 4 , two such arrays were used in training. In the first phase, either a green bottle or a red bottle appeared on a trial, with bottle identity changing at random from trial to trial (the mixed condition). The goal was to the left of the green landmark and down from the red landmark. Only control tests, with the green or red bottle alone, were given. The vectors from the red and green landmarks to the goal differed by $90^{\circ}$. If the two bottles are confused, or the vectors associated with the two bottles are confused, directional conflicts are again generated. Directional errors on these tests thus help to differentiate the two models. In later phases of the experiment, the birds were trained and tested with only the red bottle for a block of trials, and then with only the green bottle for a block (the blocked conditions). In these phases, no directional error was expected, because landmark confusion should be minimal. The hope was that the birds would search in roughly the correct direction for each bottle. The direction and radial distance of search under these no-confusion conditions are needed in order to evaluate the direction and radial distance of search caused by the confusions in the mixed condition. Thus, the blocked-trial, tests serve a purpose that was analogous to that of the control tests in Experiments 1, 2, and
3. Although training order (mixed vs. blocked) was not counterbalanced in this experiment, this is of no consequence to the testing of the two models. The crucial data are the radial distances of peak searching both with and without directional confusions.

\section{Method}

\section{Subjects}

Four pigeons experienced at searching for hidden food participated. They had not participated in any of the other experiments reported here, and they were kept under the same conditions as those in the other experiments.

\section{Apparatus}

Two different training setups were used. In one, the green bottle used in Experiment 3 was the sole landmark. The center of the goal (same as in Experiment 3) was $10 \mathrm{~cm}$ to the left of the left edge of the bottle. In the other, the red bottle used in Experiment 3 was the sole landmark, from which the goal was $10 \mathrm{~cm}$ down. Array location varied as in Experiments 1 and 3.

\section{Procedure}

Training and testing proceeded in three phases. In Phase 1, the mixed condition, both arrays were presented an equal number of times on each day. In Phase 2, only the array with the red bottle was presented. In Phase 3, only the array with the green bottle was presented. Phases 2 and 3 are called blocked conditions.

Phase 1. In Phase 1, after shaping, the birds received four trials with each bottle in a random order on $\mathbf{4}$ successive training days. The only constraint on landmark presentation was that a bottle appear no more than three times consecutively. After that, one test was given each day along with six training trials. The training trials, three with each bottle, occurred in a random order with a test scheduled for Trial 4, 5,6, or 7, chosen at random. The test array consisted of one of the bottles, but with the goal and food absent. Each test type followed a green-bottle training trial and a red-bottle training trial equally often. Four of each kind of test were given. Otherwise, the procedure and data analysis resembled those for Experiment 3. Early in testing, I piloted a number of other tests in which both landmarks were included, but the birds were disinclined to search on these tests, so these data were not analyzed. Otherwise, data analysis followed that of Experiments 1 and 3.

Phase 2. In Phase 2, only the array with the red bottle was presented. The birds were initially given 2 training days, five trials per day. Following training, a test with the goal and food absent was scheduled on each subsequent day on Trial 3, 4, or 5, chosen at random. Each bird received four such tests.

Phase 3. In Phase 3, only the array with the green landmark was presented. Seven training days were given before the tests were presented. Otherwise, the procedure followed that of Phase 2.

\section{Results and Discussion}

The results (Figure 7) showed that in the blocked tests, the birds on the whole, with the possible exception of $15 \mathrm{~B}$ with the red bottle, searched in the appropriate location for each landmark. On tests given when the two training situations were mixed within sessions, however, some of the birds searched at a location that was different from the peak place of searching on blocked-condition tests. This was true for each of the landmarks. These search peaks were displaced in the direction appropriate for the other, absent landmark. In fact, with the red bottle, all the birds showed this systematic error. The direction of search on tests from the mixed condition differed sig- 
$A$

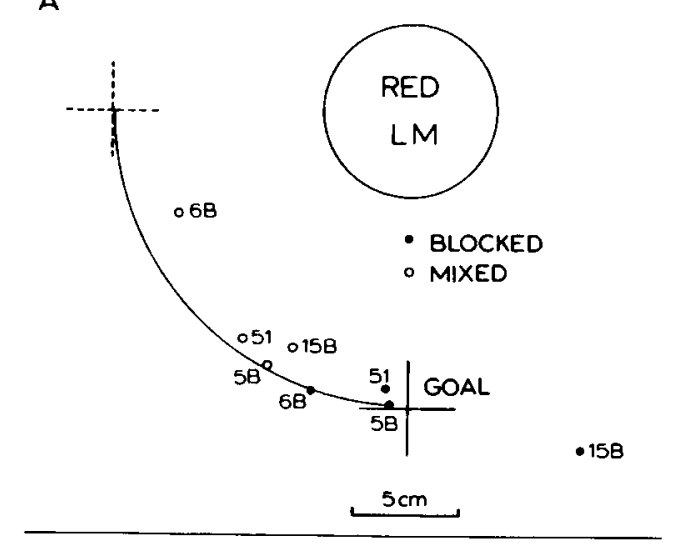

$B$

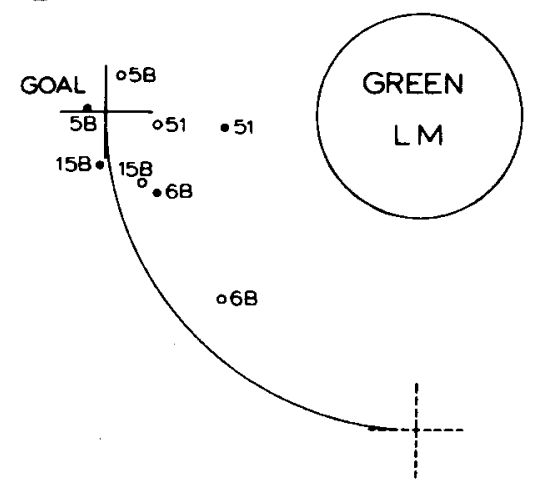

Figure 7. Places of peak searching for individual birds on tests with each landmark in Experiment 4 (A, red bottle; $B$, green bottle), when the training was blocked (always the same landmark) or mixed (either landmark might appear on a trial). The dashed crosses indicate the location of the goal according to the absent landmark.

nificantly from the direction of search on tests from the blocked condition for the red bottle $[F(1,3)=34.44]$, but not for the green bottle.

As in Experiment 3, the birds confused the two landmarks. The vectors appropriate to each were retrieved and averaged in some fashion to arrive at the final place of search. Again, the question was whether they averaged directions or entire vectors. Figure 7 shows that the radial distance of search did not change systematically on the tests in the mixed condition. Radial distances of search on mixed-training tests did not differ significantly from those on blocked-training tests for either the red or the green bottle. Again, these results favor direction averaging rather than vector averaging.

\section{MODELS}

In four experiments, pigeons were presented with two conflicting sets of cues associated with two different directions from a central point. The conflicts were generated by a new orientation of a landmark (Experiment 1), an old and inappropriate direction to the goal (Experiment 2), and by absent landmarks (Experiments 3 and 4). The experiments also differed in the nature of the search space and methods of determining the place of peak searching. In some cases in each experiment, the pigeons compromised between the two directions dictated by the two sets of cues. Two different models make different predictions on how the dictates of the two sets of cues were averaged. The data from all four experiments together provide the best test to differentiate between the two models. I include all data in the following analyses, even those that do not discriminate between the two models, because the latter help to provide additional estimates in curve fitting.

According to the vector-averaging model, the radial distance of search is shorter when an intermediate direction of search is chosen. Its prediction is given by the formula for $|\mathbf{x}|$ in Figure 2. This prediction follows if the bird is averaging two vectors. If the bird is averaging only the directional component of the vectors, however, and determining radial distance independently from direction, then it should search at the same radial distance, regardless of direction. In Figure 8, the predictions of the two models are plotted against the observed radial distance data from the experimental tests. For the directionaveraging model, each subject's predicted radial distance of search on experimental tests is its radial distance of search on control tests. For the vector-averaging model, each subject's predicted radial distance of search is given by the formula for $|\mathbf{x}|$ in Figure 2,

$$
|\mathbf{x}|=\frac{|\mathbf{u}| \cos (\phi / 2)}{\cos (\phi / 2-\vartheta)}
$$

where $|\mathbf{u}|$ is its radial distance of search on control tests, $\phi$ is the angle between the two conflicting sources of information $\left(90^{\circ}\right.$ in all the experiments), and $\vartheta$ is the angular difference between its directions of search on control and experimental tests. The difference is signed; it is positive if the direction on experimental tests is closer to the direction dictated by the conflicting cue. All negative values of $\vartheta$, however, were assigned the value of $0^{\circ}$ in calculating the predicted distance of the vectoraveraging model. Similarly, values of $\vartheta$ greater than $90^{\circ}$ were assigned the value of $90^{\circ}$. That is, when the experimental direction of search lies in the "wrong" direction $\left(\vartheta<0^{\circ}\right)$ or differs from the control direction by more than the extent of the directional conflict $\left(\vartheta>90^{\circ}\right)$, the vector-averaging model was taken to predict the same radial distance of search on experimental and control tests, making predictions identical to those of the direction-averaging model. In Figure 8, data points from all the experiments are shown ( 3 from Experiment 1, 4 from Experiment 2, and 8 each from Experiments 3 and 4 for a total of 23). The filled dots represent data points on which the vector-averaging model predicts a value that is $85 \%$ or less of the predicted value of the directionaveraging model. The dotted line represents the predicted values of each model, neither of which has any free parameters.

Inspection of Figure 8 shows that the data points are unsystematically scattered about the predicted values for 
the direction-averaging model, whereas they seem to lie systematically above the predicted values for the vectoraveraging model. This is especially evident in the data points for which the prediction of the vector-averaging model is $85 \%$ or less than the prediction of the directionaveraging model. Statistical evidence supports these impressions in four ways.

First, best-fitting regression slopes with the $y$ intercept at 0 (least squared criterion) were found for each model (solid lines in Figure 8). To obtain the best estimate, all the data were included. This slope should be 1 if the model is correct. The best-fitting slope was 0.97 for the direction-averaging model, and 1.17 for the vectoraveraging model.

Second, fitting data to a model should reduce the variance in the observed data. I estimated the performance of the two models by comparing the sums of squared deviations of observed data points from the predictions of each model against the sums of squared deviations from the mean of the observed data. The latter are, of course, the deviations used to calculate variance in the data, and the comparisons provide a measure of how each model reduces variance in the data. The direction-averaging model reduced the sums of squared deviations by $38 \%$, whereas the vector-averaging model actually increased the sums of squared deviations by $17 \%$. In this context, the variance reduction is equivalent to percent variance accounted for. The $38 \%$ reduction provided by the direction-averaging model should not be disparaged, given the restricted range in the observed data (the radial distance of the goal was similar in all experiments) and the fact that the model has no free parameters. A regression line fitted to the data in the right panel of Figure 8 (with two free parameters of slope and intercept) only accounted for $42 \%$ of the variance. The directionaveraging model thus improves the fit in the data from the prediction of the mean observed radial distance of search, but the vector-averaging model does not.

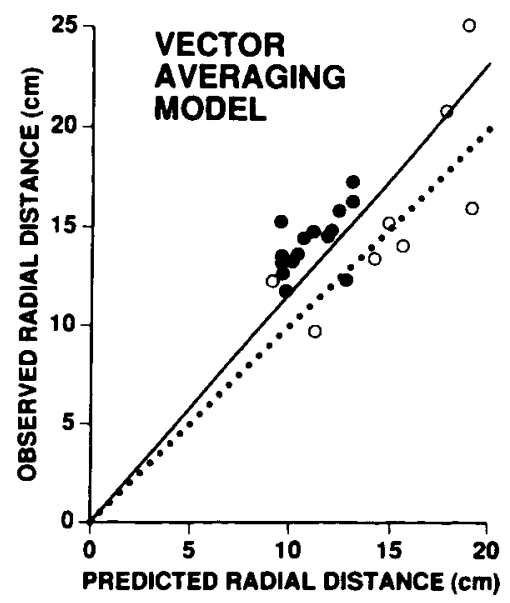

For the next two analyses, two different sets of data were examined for each model: (1) the entire data set in Figure 8, and (2) a selected data set in which the data points are all independent. Set 2 was chosen in order to meet the assumptions of the statistical tests (regression analysis and $t$ test) used. Included in the set are data from Experiment 1, Experiment 2, the green-bottle and control tests from Experiment 3, and the red-bottle tests from Experiment 4. In this way, each bird contributes only one data point. Data selected were those for which predictions of the two models differed the most. The selection was in no way biased in favor of either model.

Third, regression lines with slope and intercept as free parameters were fitted to Data Sets 1 and 2. These allow an estimation of the range in which the predicted values should fall. Regression lines are useful because $95 \%$ confidence intervals can be readily estimated. If the predictions of the models are correct, the $95 \%$ confidence intervals about the predicted radial distance based on the regression lines ought to include the predicted radial distance based on the models. The $95 \%$ confidence interval about the predicted dependent variable is smallest at the mean. At the mean radial distance of search on experimental tests in each data set, the $95 \%$ confidence interval of the prediction based on the regression line includes the predicted value in the case of the directionaveraging model, but not in the case of the vectoraveraging model. Thus, the vector-averaging model underpredicts the observed data, but the direction-averaging model is consistent with the data.

Fourth, the predicted values were compared against the observed radial distances of search for both models, using paired $t$ tests. In both data sets, predicted and observed values differed significantly for the vectoraveraging model $[t(22)=4.79$ for Data Set 1 , and $t(15)=$ 8.66 for Data Set 2], but not for the direction-averaging model. Thus, errors are systematic for the vectoraveraging model, but are unsystematic for the direction-

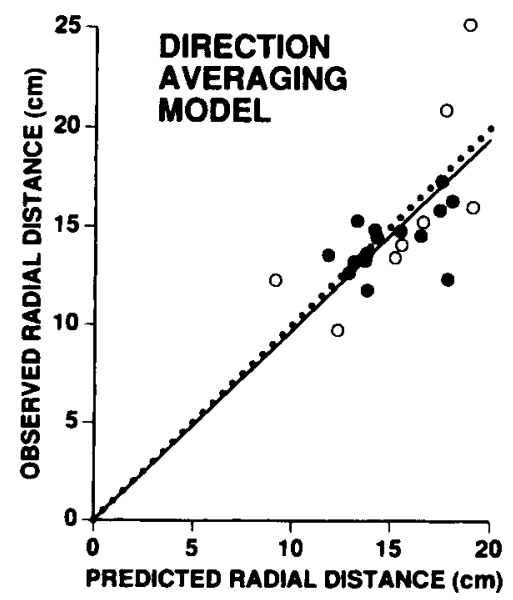

Figure 8. Observed radial distance of the place of peak searching on experimental tests plotted against the predicted radial distance for the vector-averaging and direction-averaging models. The dotted lines represent a perfect fit between the data and the model. The solid lines represent the best-fitting linear function through the origin by the least squares criterion. See text. 
averaging model. On these bases, the direction-averaging model cannot be rejected, but the vector-averaging model can.

\section{GENERAL DISCUSSION}

In conclusion, the data from these directional-conflict experiments allow us to reject the vector-averaging model as an account of how pigeons make compromises between different directions. The birds were not averaging entire vectors pointing in different directions, but only their directional components. I suggest that distance is computed independently, which results in the birds' searching at the same radial distance in experimental and control conditions.

Note that when an intermediate direction of search was found on an experimental test, a single-peaked search distribution was found. This means that the birds could not be using only one set of cues (e.g., unshifted landmarks) on some tests and only another set (e.g., the rotated landmark) on other tests, because such a hypothesis would predict double-peaked search distributions. An intermediate direction of search is also not predicted if the birds only used the cues pointing to the original goal direction and were simply less sure of where to search on experimental tests (because the test condition looked different from the usual). Such a generalization decrement hypothesis would predict a larger scatter in the search, but not a change in the average search direction. The most plausible hypothesis, then, is that the birds were averaging the directional dictates of different cues.

The fact that the various manipulations of directional cues in these experiments all induced at least some of the pigeons to change their direction of search implies that no one source of directional cue is predominant in all pigeons. Rather, different factors are weighted and averaged, and the weighting differs from bird to bird and from situation to situation. Experiment 1 suggests that the orientation of a local feature (the strip on the bottle) is used, in part, in determining direction. In unpublished data, Fiset and Cheng (1991) have also found that the direction of ambient light in the room is used, as well as the orientation of a larger tray surrounding the search tray (a local frame of geometry). In addition, since the birds did not always follow the manipulated cue completely, the unmanipulated directional cues must have been used as well. These unmanipulated cues include the geometry of the room at large, notable features in the room (such as the observation window/door), magnetic cues, and inertial cues. It has been shown that birds use inertial cues (von St. Paul, 1982) and perhaps magnetic cues (Keeton, 1974), although the use of magnetic cues in laboratory settings has not been found (Alsop, 1987; Carman, Walker, \& Lee, 1987; McIsaac \& Kreithen, 1987; Moore, Stanhope, \& Wilcox, 1987). Rats show evidence of using global geometry in determining direction (Cheng, 1986; Margules \& Gallistel, 1988).

As for the determination of distance, neither a change in width nor a change in height affects the distance of search in pigeons (Cheng, 1988), suggesting that the projected retinal size does not play a large role. The true landmark-to-goal distance is somehow extracted and used.

As in directional determination, I suggest that pigeons average distance cues from different sources. An example comes from experiments by Cheng and Sherry (1992), in which the goal was near one straight edge but far from other straight edges. Another object near the goal served as a landmark. When the landmark was moved a small distance parallel to the edge, the birds (pigeons and chickadees) shifted their peak searching in the same direction and by the same amount. They were apparently using only the landmark to determine where to search along the dimension parallel to the edge. But when the landmark was shifted to be perpendicular to the edge, the birds shifted their peak searching by various amounts perpendicular (but not parallel) to the edge. Cheng and Sherry proposed that the perpendicular distance of the goal from the edge was used in part in the birds' calculations. Measuring a perpendicular distance means measuring a particular distance in a particular direction (perpendicular from the edge) from some starting point along the edge. The starting point is apparently determined by the position of the landmark along the parallel dimension in these experiments. The distance is determined by a compromise between the dictates of the position of the landmark (along the perpendicular dimension) and the edge. According to this interpretation, the birds were maintaining the same direction (perpendicular to the edge) but compromising on the distance.

Despite the fact that I have rejected the vectoraveraging model, or what I have previously called the vector sum model (Cheng, 1989), the similarity between the direction-averaging and vector sum models should not be overlooked. Like the vector sum model, in the direction-averaging model the bird is said to encode vectors - that is, distances and directions from landmarks to the goal. Multiple sources are used in computing where to search. The computation takes the form of averaging the dictates of different sources of information. The difference is that the vector sum model averages vectors in their entirety, but the direction-averaging model averages components of vectors, directions, and distances independently.

In its computational operations, the direction-averaging model is also similar to a model of landmark-based spatial search developed for honeybees (Cartwright \& Collett, 1982, 1983). In the honeybee model, the bee encodes a template of what the nearby landmark array looks like from the vantage point of the goal. The wholistic representation connoted by the term "template" is misleading, however, because computations are performed on individual elements of the template, each independently of the others. The elements are taken to be the landmarks (black cylinders used in the experiments) and the spaces between them. The computations based on each element are then averaged to determine a direction of movement. From each element, two movement vectors are computed. These movement vectors are direc- 
tional vectors; they contain no distance component, and they serve only to point to the direction in which the bee is to fly.

One movement vector is based on distance information. It is based on a comparison of the retinal image size of the perceived element and its expected or correct size on the template. The vector serves to move the bee closer to landmarks that look too small, and farther from landmarks that look too large. The other vector is based on directional information. It is based on a comparison of the compass direction of the perceived landmark and its counterpart on the template. It serves to turn the bee to the right or left to align the compass direction of the perceived landmark with its correct compass direction according to the template. Thus, like the model for pigeons, calculations of distances and directions go on independently, and the results of all calculations are then averaged in guiding behavior.

In spatial search, one other example of a directional compromise based on multiple sources comes from work on hamsters by Etienne and colleagues (Etienne, Lambert, Reverdin, \& Teroni, 1993; Etienne, Teroni, Hurni, \& Portenier, 1990). In these experiments, the hamster ventured from its home at the edge of the arena to the center to find food to hoard. In some conditions, a single light permanently positioned over the home was rotated, putting landmark-based directional information in conflict with inertial sense of direction. Some hamsters compromised by heading in an intermediate direction between the directional dictates of the two sources.

I suspect that this scheme of independent calculations followed by averaging is common in animals. As I pointed out with respect to the vector sum model (Cheng, 1988,1989 ), this scheme is advantageous whenever the use of multiple sources of information can improve the accuracy of calculations. The modularity of computational units makes the calculations based on different sources independent statistically and is readily realized neurophysiologically. The outputs of computational units need only to feed independently into a summator, the amplitude of their outputs modulated by other units that serve as weighting factors. In this way, the particular models of landmark-based spatial search developed thus far might be revealing some general principles of neurocognitive architecture.

\section{REFERENCES}

Alsop, B. (1987). A failure to obtain magnetic discrimination in the pigeon. Animal Learning \& Behavior, 15, 110-114.

BATSCHELET, E. (1981). Circular statistics in biology. New York: Academic Press.

Carman, G. J., Walker, M. M., \& Lee, A. K. (1987). Attempts to demonstrate magnetic discrimination by homing pigeons in flight. Animal Learning \& Behavior, 15, 124-129.

Cartwright, B. A., \& Collett, T. S. (1982). How honeybees use landmarks to guide their return to a food source. Nature, 295, 560-564.

CARTwright, B. A., \& Collett, T. S. (1983). Landmark learning in bees. Journal of Comparative Physiology A, 151, 521-543.
CHENG, K. (1986). A purely geometric module in the rat's spatial representation. Cognition, 23, 149-178.

Cheng, K. (1988). Some psychophysics of the pigeon's use of landmarks. Journal of Comparative Physiology A, 162, 815-826.

CHENG, K. (1989). The vector sum model of pigeon landmark use. Journal of Experimental Psychology: Animal Behavior Processes, 15, 366-375.

ChEnG, K. (1990). More psychophysics of the pigeon's use of landmarks. Journal of Comparative Physiology A, 166, 857-863.

CHENG, K., \& SHERRY, D. F. (1992). Landmark-based spatial memory in birds: The use of edges and distances to represent spatial positions. Journal of Comparative Psychology, 106, 331-341.

Collett, T. S., Cartwright, B. A., \& SMITh, B. A. (1986). Landmark learning and visuo-spatial memories in gerbils. Journal of Comparative Physiology A, 158, 835-851.

DYER, F. C., \& GouLD, J. L. (1983). Honeybee navigation. American Scientist, 71, 587-597.

Etienne, A. S., Lambert, S. J., Reverdin, B., \& Teroni, E. (1993). Learning to recalibrate the role of dead reckoning and visual cues in spatial navigation. Animal Learning \& Behavior, 21, 266-280.

Etienne, A. S., Teroni, E., Hurni, C., \& Portenier, V. (1990). The effect of a single light cue on homing behaviour of the golden hamster. Animal Behaviour, 39, 17-41.

FISET, S., \& CHENG, K. (1991). [Directional determination in pigeons.] Unpublished raw data.

Gallistel, C. R. (1990). The organization of learning. Cambridge, MA: MIT Press.

KEETON, W. T. (1974). The orientation and navigational basis of homing in birds. In D. Lehrman, R. Hinde, \& E. Shaw (Eds.), Advances in the study of behavior (Vol. 5, pp. 47-132). New York: Academic Press.

Margules, J., \& Gallistel, C. R. (1988). Heading in the rat: Determination by environmental shape. Animal Learning \& Behavior, 16, 404-410.

McIsaAc, H. P., \& Kreithen, M. L. (1987). Attempts to condition homing pigeons to magnetic cues in an outdoor flight cage. Animal Learning \& Behavior, 15, 118-123.

Moore, B. R., Stanhope, K. J., \& Wilcox, D. (1987). Pigeons fail to detect low-frequency magnetic fields. Animal Learning \& Behavior, 15, 115-117.

O'KeEFe, J., \& ConwaY, D. H. (1980). On the trail of the hippocampal engram. Physiological Psychology, 8, 229-238.

ROBERTS, S. (1981). Isolation of an internal clock. Journal of Experimental Psychology: Animal Behavior Processes, 7, 242-268.

Spetch, M. L., Cheng, K., \& Mondloch, M. V. (1992). Landmark use by pigeons in a touch-screen spatial search task. Animal Learning \& Behavior, 20, 281-292.

Spetch, M. L., \& Edwards, C. A. (1988). Pigeons' (Columba livia) use of global and local cues for spatial memory. Animal Behaviour, 36, 293-296.

Suzuki, S., Augerinos, G., \& Black, A. H. (1980). Stimulus control of spatial behavior on the eight-arm maze in rats. Learning \& Motivation, 11, 1-18.

Tinbergen, N. (1972). The animal in its world. Cambridge, MA: Harvard University Press.

VANDER WALL, S. B. (1982). An experimental analysis of cache recovery in Clark's nutcracker. Animal Behaviour, 30, 84-94.

voN FrIsCH, K. (1953). The dancing bees (D. Ilse, Trans.). San Diego: Harcourt Brace Jovanovich.

VON ST. PAUL, U. (1982). Do geese use path integration for walking home? In F. Papi \& H. G. Wallraff (Eds.), Avian navigation (pp. 297307). Berlin: Springer-Verlag.

WEHNER, R., \& RAEBER, F. (1979). Visual spatial memories in desert ants, Cataglyphis bicolor (Hymenoptera: Formicidae). Experientia, 35, 1569-1571.

(Manuscript received July 26, 1993; revision accepted for publication December 14, 1993.) 